\title{
Darstellung aromatischer Fluor-Verbindungen durch nucleophilen Austausch von Nitro-Gruppen gegen Fluorid
}

\author{
Franz Effenberger * und Willi Streicher ${ }^{1 \text { ) }}$ \\ Institut für Organische Chemie der Universität Stuttgart, \\ Pfaffenwaldring 55, D-7000 Stuttgart 80
}

Eingegangen am 16. Mai 1990

Key Words: Fluoro compounds, aromatic / Nitrite exchange by fluoride

\section{Synthesis of Aromatic Fluoro Compounds by Nucleophilic Exchange of Nitro Groups by Fluoride}

The synthesis of aromatic fluoro compounds from the respective nitro compounds by nucleophilic substitution of nitrite by fluoride is described. Reasonable yields in case of nonactivated nitro compounds are only obtained if the nitrite formed in the reaction is eliminated by acylation. 1-Fluoro-3-nitrobenzene (2) was obtained from 1,3-dinitrobenzene (1), and 1fluoro-3,5-dinitrobenzene (9) as well as 1,3-difluoro-5-nitroben- zene (10) from 1,3,5-trinitrobenzene (8) in yields up to $92 \%$ by reaction of nitro compounds with potassium fluoride in sulfolane at $180-200^{\circ} \mathrm{C}$ in the presence of phthaloyl dichloride (6); 1,2-difluoro-4-nitrobenzene (12) was formed in $58 \%$ yield from 2,4-dinitro-1-fluorobenzene (11) in the presence of pyromellitoyl tetrachloride (13).
Die immer noch wichtigste und vielseitigste Methode zur Darstellung aromatischer Fluor-Verbindungen ist die Thermolyse von Aryldiazonium-tetrafluoroboraten nach Balz-Schiemann ${ }^{2)}$. Da die Diazonium-Salze in der Regel, ausgehend von Nitro-Verbindungen, über die entsprechenden Amine und deren Diazotierung erhalten werden, und die Thermolyse der Diazonium-Salze oft nur mit mäBigen Ausbeuten abläuft ${ }^{2)}$, wäre ein direkter Austausch von NitroGruppen gegen Fluor - ohne den Umweg über DiazoniumSalze - von großem präparativen Nutzen.

Im Rahmen allgemeiner Untersuchungen zur nucleophilen Aromaten-Substitution ${ }^{3)}$ stellte sich in den letzten Jahren immer deutlicher heraus, daß Nitrit bei solchen Reaktionen eine sehr gute Austrittsgruppe ist und es in manchen Fällen sogar die Austrittstendenz des Fluorids übertrifft ${ }^{3 c, 4)}$. Zusammenfassungen über den nucleophilen Austausch aromatischer Nitro-Gruppen sind erschienen ${ }^{5}$, wobei Beck ${ }^{5 a)}$ umfassend auch auf den Austausch von nicht in ortho- bzw. para-Stellung aktivierten Nitro-Gruppen eingeht. Trotz guter Kenntnisse der Substitution aromatischer Nitro-Gruppen durch Fluorid sind bisher nur wenige Beispiele der Anwendung dieser Reaktion zur Darstellung aromatischer Fluor-Verbindungen beschrieben. Ein Grund dafür könnten die zum Teil niedrigen Ausbeuten $(10-45 \%)$ an Fluor-Verbindungen sein, die - auch in dipolar-aprotischen Lösungsmitteln bei hohen Reaktionstemperaturen und langen Reaktionszeiten - erhalten werden ${ }^{6,7)}$ und die mit Nebenreaktionen infolge Zersetzung des Lösungsmittels ${ }^{8)}$ sowie der Bildung von Phenolen und Phenolethern durch HydrolyseReaktionen ${ }^{6)}$ begründet werden. Besonders auffallend - und bisher ohne plausible Erklärung - ist die Diskrepanz der in der Literatur beschriebenen Reaktionen von $m$-Dinitrobenzol: Während mit Alkoholaten und Thiolaten in dipolar-aprotischen Lösungsmitteln Monosubstitutionsprodukte mit bis zu $90 \%$ Ausbeute isoliert werden konnten ${ }^{9}$, wurden mit Fluorid, selbst in Hexamethylphosphortriamid (HMPA), maximal $45 \% \mathrm{~m}$-Fluornitrobenzol erhalten ${ }^{7}$. Da wir an der Herstellung speziell substituierter Fluoraromaten interessiert waren, haben wir den nucleophilen Austausch von Nitrit gegen Fluorid in Aromaten grundlegend untersucht.
Nucleophile Substitution von Nitrit an 1,3-Dinitrobenzol (1) durch Fluorid

Bei der Umsetzung von 1,3-Dinitrobenzol (1) mit überschüssigem Kaliumfluorid in $N$-Methylpyrrolidon erhielten wir, je nach Reaktionsbedingungen, wechselnde Mengen 1Fluor-3-nitrobenzol (2) und 3,3'-Dinitrodiphenylether (5).

Schema 1<smiles>O=[N+]([O-])c1cccc([N+](=O)[O-])c1</smiles>
2 3<smiles>COc1cccc([N+](=O)[O-])c1</smiles>

Schon Finger und Kruse ${ }^{6}$ hatten die Bildung von Diphenylethern bei der Darstellung von 1-Fluor-2(4)-nitro4(2)-(trifluormethyl)benzolen aus den entsprechenden 1Chlor-Verbindungen mit Kaliumfluorid gefunden; sie erklärten diese Reaktion über eine teilweise Hydrolyse der Chlor- oder Fluor-Verbindung zum entsprechenden Phenol, das dann mit einem weiteren Molekül Halogen-Verbindung den Diphenylether bildet. Bei Verwendung von Kaliumfluorid mit unterschiedlichem Wassergehalt beobachteten wir in 
unseren Versuchen jedoch keine Änderung des Produktverhältnisses 2/5, d. h. eine direkte Hydrolyse der Fluor-Verbindung $\mathbf{2}$ ist wenig wahrscheinlich. Dagegen ist die Bildung des Diphenylethers über die Weiterreaktion des primär entstehenden Produktes 2 mit Kaliumnitrit leicht zu deuten: Kaliumnitrit kann als ambidentes Nucleophil auch zum Salpetrigsäureester 3 reagieren und dieser mit weiterem $\mathrm{Ka}$ liumnitrit unter $\mathrm{N}_{2} \mathrm{O}_{3}$-Abspaltung Kaliumphenolat 4 bilden. Letzteres kann mit 2 den isolierten Diphenylether 5 bilden. DaB Nitrit mit gleicher Wahrscheinlichkeit Reaktionen am $\mathrm{O}$ - wie am N-Atom eingeht, zeigen ausführliche kinetische Untersuchungen ${ }^{10)}$ der Umsetzungen zahlreicher aktivierter Halogenaromaten mit Nitrit in dipolar-aprotischen Lösungsmitteln. Einen weiteren Beweis für den in Schema 1 aufgeführten Reaktionsablauf erbrachte die Umsetzung der isolierten Monofluor-Verbindung 2 mit molaren Mengen Kaliumnitrit bei $170^{\circ} \mathrm{C}$ in $\mathrm{N}$-Methylpyrrolidon, die zu einem Gemisch aus 1, 2 und 5 führte. Da unter diesen Bedingungen aus 1 mit Kaliumnitrit kein Diphenylether 5 gebildet wird, kann man davon ausgehen, daß 4 bevorzugt mit 2 und nicht mit 1 zu 5 reagiert (Schema 1).

Nach den voranstehend beschriebenen Ergebnissen sollten höhere Ausbeuten an Fluoraromat 2 erhalten werden, wenn das beim Austausch entstehende Nitrit abgefangen wird, um dessen Weiterreaktion zu 3 zu unterbinden. Die für eine Nitrit-Entfernung beschriebenen Methoden - Oxidations- bzw. Reduktionsverfahren oder Umsetzungen mit geeigneten Elektrophilen - sind jedoch zum größten Teil ungeeignet, da die hierbei erforderlichen hohen Reaktionstemperaturen stören und zum Teil auch Edukte bzw. Produkte angegriffen werden. So werden bei den meisten Reduktionsverfahren auch die aromatischen Nitro-Gruppen reduziert; lediglich Reduktionen mit Harnstoffen oder Ammonium-Salzen bilden eine Ausnahme. Da Harnstoff jedoch auch als Nucleophil reagiert, kommen für eine reduktive Entfernung von Nitrit nur Ammonium-Salze in Frage.

Nachdem Vorversuche zeigten, daB Ammoniumsulfat in $\mathrm{N}$-Methylpyrrolidon bis $190^{\circ} \mathrm{C}$ stabil ist und bei Zugabe von Kaliumnitrit bei $170^{\circ} \mathrm{C}$ Stickstoff abgespalten wird, führten wir die Umsetzung von 1 mit Kaliumfluorid/Cäsiumfluorid in Gegenwart von Ammoniumsulfat durch. Nach sechsstündigem Erhitzen auf $170^{\circ} \mathrm{C}$ wurde jedoch nur 3-Nitroanilin in 13proz. Ausbeute isoliert.

Als weitere Möglichkeit zur Nitrit-Entfernung haben wir die oben beschriebenen Umsetzungen in Gegenwart von Elektrophilen untersucht. Da man mit Alkylierungs-Mitteln in Gegenwart von überschüssigem Kaliumfluorid bevorzugt mit der Bildung von Alkylfluoriden rechnen muß, haben wir im wesentlichen Acylierungs-Mittel eingesetzt, die primär zu Acylnitriten und dann mit weiterem Nitrit unter $\mathrm{N}_{2} \mathrm{O}_{3}$-Austritt irreversibel zu Carboxylaten reagieren. Eine Nitrit-Entfernung mit einem Acylierungs-Mittel wurde beim Austausch der 4-Nitro-Gruppe in 4-Nitrophthalsäureanhydrid mit Kaliumfluorid beobachtet und bewiesen, wobei das Edukt gleichzeitig als Acylierungsmittel wirkt ${ }^{111}$; da es sich hierbei jedoch um die Reaktion einer aktivierten NitroGruppe handelt, kommt in dem beschriebenen Beispiel der Nitrit-Entfernung nicht die entscheidende Bedeutung zu.
Wir haben als Acylierungs-Agens für die Nitrit-Entfernung Phthaloyldichlorid (6) eingesetzt, das einen für diese Reaktionen ausreichend hohen Siedepunkt $\left(281^{\circ} \mathrm{C}\right)$ besitzt und thermisch weitgehend stabil ist. Erhitzt man 6 mit Kaliumnitrit in Sulfolan als Lösungsmittel auf $180^{\circ} \mathrm{C}$, so entwickeln sich Stickoxide unter Bildung von Phthalsäureanhydrid (7), nach 2.5 Stunden ist kein Nitrit mehr nachweisbar.

Schema 2

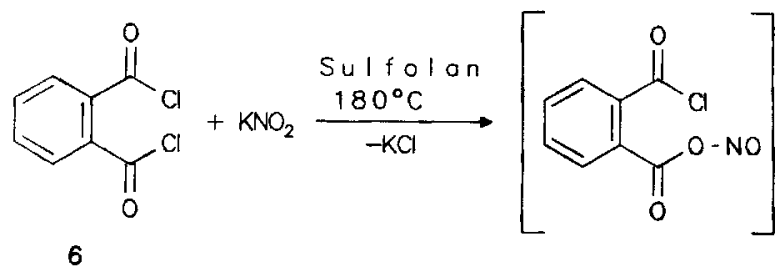<smiles>O=C1O[C+]([N+](=O)[O-])C(=O)c2ccccc21</smiles>

7

Tab. 1. Umsetzung von 1,3-Dinitrobenzol (1) mit KF in Gegenwart von Phthaloyldichlorid (6) bei $200^{\circ} \mathrm{C}$ in Sulfolan zu 1-Fluor-3-nitrobenzol (2)

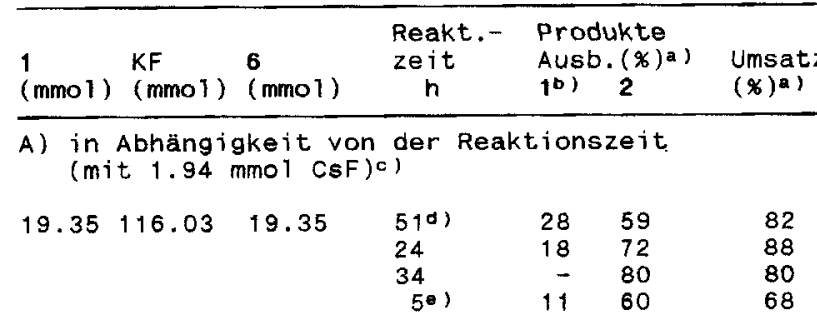

B) in Abhängigkeit von der Reaktionszeitc)

$\begin{array}{lllllll}19.35 & 96.72 & 19.37 & 12 & 77 & 22 & 95 \\ & & & 24 & 57 & 40 & 93 \\ & & 36 & 42 & 50 & 86 \\ & & 72 & 17 & 65 & 80\end{array}$

C) in Abhängigkeit von der Reaktionszeit (portionsweise Zugabe von $6 \mathrm{zu}$ Beginn der Reaktion, nach $24 \mathrm{~h}$ und nach $48 \mathrm{~h}$ jeweils bei $140^{\circ} \mathrm{C}$ )

$19.35 \quad 96.72 \quad 29.02$

$\begin{array}{llll}12 & 77 & 22 & 95 \\ 24 & 57 & 40 & 93 \\ 36 & 41 & 55 & 93 \\ 48 & 30 & 65 & 93 \\ 72 & 17 & 75 & 90\end{array}$

D) in Abhängigkeit von der Menge an KFo)

\begin{tabular}{rrrrrrr}
19.35 & 77.24 & 29.02 & 72 & 40 & 56 & 93 \\
96.72 & & & 17 & 75 & 91 \\
116.03 & & & 17 & 75 & 91 \\
\hline
\end{tabular}

a) Gaschromatographisch bestimmt. - b) Nicht umgesetztes 1. c) Einmalige Zugabe molarer Mengen 6 zu Beginn der Reaktion. d,e) Reaktionstemperatur: d) $190^{\circ} \mathrm{C}$, e) $^{\circ} 220^{\circ} \mathrm{C}$. 
Die in Tab. 1 aufgeführten Ergebnisse unserer Umsetzungen von $m$-Dinitrobenzol (1) mit Kaliumfluorid in Gegenwart von 6 unter vielseitiger Variation der Reaktionsbedingungen zeigen, daß auf diese Weise die Fluor-Verbindung 2 in Ausbeuten bis zu $80 \%$ entsteht und ein maximaler Umsatz von über $90 \%$ erreicht werden kann.

In präparativen Ansätzen konnten wir 2 mit $70 \%$ Ausbeute isolieren. Das bei der Reaktion gebildete Phthalsäureanhydrid (7) fällt dabei in sehr reiner Form an (Ausb. 78\%) und kann direkt mit Phosphorpentachlorid wieder in das Dichlorid 6 übergeführt werden.

\section{Nucleophile Substitution von Nitrit durch Fluorid in 1,3,5-Trinitrobenzol (8), 2,4-Dinitrofluorbenzol (11) und 4,4'-Dinitrobiphenyl (14)}

Nachdem in Gegenwart von Phthaloyldichlorid (6) eine erhebliche Ausbeutesteigerung beim Nitrit-Austausch in 1,3Dinitrobenzol (1) durch Fluorid erzielt werden konnte, haben wir untersucht, ob diese Methode auch bei weiteren Nitro-Verbindungen angewendet werden kann. Bei 1,3,5Trinitrobenzol (8) gelang uns ein sukzessiver Austausch von zwei Nitro-Gruppen gegen Fluorid unter Zugabe der doppelt molaren Menge 6 in Sulfolan bei $180^{\circ} \mathrm{C}$. Nach 2 Stunden setzte die Reaktion ein, ergab nach insgesamt 140 Minuten selektiv 1-Fluor-3,5-dinitrobenzol (9) in 92proz. Ausbeute und nach insgesamt 12stdg. Reaktionszeit 77\% 1,3-Difluor-5-nitrobenzol (10) (Schema 3, Tab. 2). Unter gleichen Bedingungen, jedoch mit nur molaren Mengen 6 setzte die Reaktion schon nach 33 Minuten ein und führte nach insgesamt 52 Minuten selektiv zum Monosubstitutionsprodukt $9(93 \%)$. Ohne Zugabe von 6 erfolgte eine Reaktion nach zwei Minuten, jedoch wurde 9 nur in einer maximalen Ausbeute von $41 \%$ erhalten. Im weiteren Reaktionsverlauf nahm dann die Ausbeute an 9 wieder ab; nach 30 Minuten konnte keine Ausgangsverbindung mehr, sondern nur noch $9(4 \%)$ sowie das Disubstitutionsprodukt 10 $(8 \%)$ nachgewiesen werden.

Schema 3<smiles>O=[N+]([O-])c1cc([N+](=O)[O-])cc([N+](=O)[O-])c1</smiles>

8

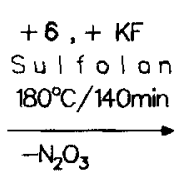

Sulfolan $-\mathrm{N}_{2} \mathrm{O}_{3}$ $\mathrm{O}_{2}$<smiles>O=[N+]([O-])c1cc(F)cc([N+](=O)[O-])c1</smiles>

9

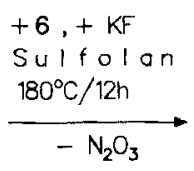<smiles>O=[N+]([O-])c1cc(F)cc(F)c1</smiles>

10

Die auf diese Weise auch in präparativem Maßstab mit $85 \%$ bzw. $70 \%$ Ausbeute leicht zugänglichen FluornitroVerbindungen 9 und 10 waren bisher nur sehr viel aufwendiger zugänglich. Die Monofluor-Verbindung 9 wurde aus
Tab. 2. Umsetzung von 1,3,5-Trinitrobenzol (8) mit KF bei $180^{\circ} \mathrm{C}$ in Sulfolan zu 1-Fluor-3,5-dinitrobenzol (9) und 1,3-Difluor-5-nitrobenzol (10)

Methode A: mit 2 mol Phthaloyldichlorid (6)/mol 8; Methode B: mit $1 \mathrm{~mol} \mathrm{6/mol} \mathrm{8;} \mathrm{Methode} \mathrm{C:} \mathrm{ohne} 6$

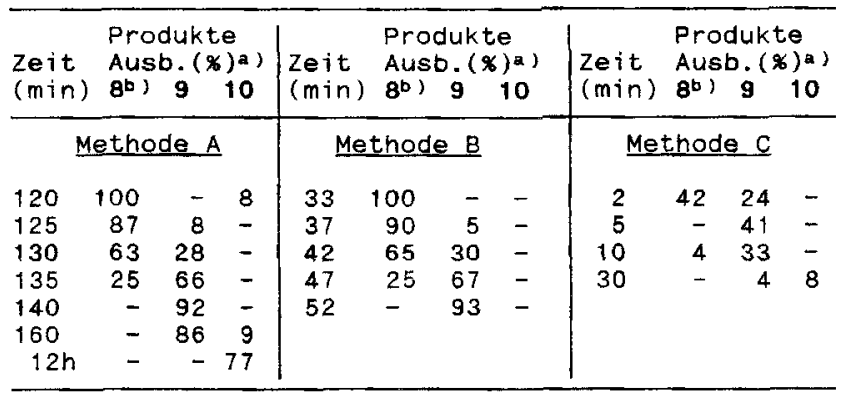

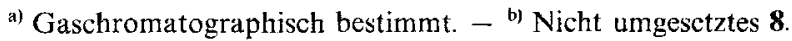

3,5-Dinitrobenzoldiazonium-tetrafluoroborat nach BalzSchiemann in nur 15 proz. Ausbeute ${ }^{12\}}$ erhalten und die Difluor-Verbindung 10, ausgehend von $m$-Phenylendiamin über fünf Reaktionsstufen in einer Gesamtausbeute von nur $10 \%{ }^{13)}$.

Untersuchungen zur Darstellung von 1,2-Difluor-4-nitrobenzol (12) aus dem leicht zugänglichen 2,4-Dinitrofluorbenzol ${ }^{14)}$ (11) haben wir zum einen wegen der Bedeutung von 12 als Ausgangsbasis für ein Pflanzenschutzmittel ${ }^{15}$ ) durchgeführt, zum anderen stellte sich die Frage der Selektivität des Nitrit/Fluorid-Austauschs bei zwei vergleichbar aktivierten Nitro-Gruppen.

Schema 4

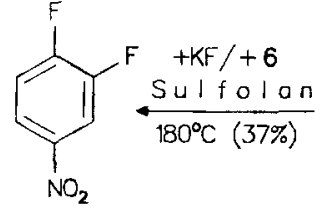

12<smiles>O=[N+]([O-])c1ccc(F)c([N+](=O)[O-])c1</smiles>

11 13:<smiles>O=C(Cl)c1cc(C(=O)Cl)c(C(=O)Cl)cc1C(=O)Cl</smiles>

Die bisher in der Literatur beschriebenen Synthesen für 12 verlaufen über 5-7 Stufen und ergeben dann lediglich Gesamtausbeuten zwischen 5 und $12 \%{ }^{16,177}$. Bei der Umsetzung von 11 mit Kaliumfluorid in Dimethylformamid haben Finger und Kruse $^{6)}$ trotz langer Reaktionszeiten $(16 \mathrm{~h}$ bei $145^{\circ} \mathrm{C}$ ) die Difluor-Verbindung 12 in nur 10proz. Ausbeute als Rohprodukt erhalten.

Wir konnten jetzt unter den voranstehend beschriebenen Bedingungen, d.h. Zugabe von molaren Mengen 6 in Sulfolan als Lösungsmittel, die Ausbeute an 12 zwar steigern, die erhaltenen $37 \%$ sind jedoch für ein präparatives Verfahren immer noch ungenügend. Der Grund für die im Ver- 
gleich zur Darstellung von $\mathbf{1 0}$ deutlich niedrigere Ausbeute ist in der großen Reaktivität von 11 gegenüber Nucleophilen zu sehen; austretendes Nitrit kann mit 11 rasch zu 2,4-Dinitrophenol reagieren, das bei diesen Umsetzungen tatsächlich als Nebenprodukt anfällt. Durch Zugabe des im Vergleich zu Phthaloyldichlorid (6) noch reaktiveren und ebenfalls leicht zugänglichen Pyromellitoyltetrachlorids (13), das wirkungsvoller als 6 in Konkurrenz zur Reaktion von Nitrit mit 11 tritt, gelang uns eine Ausbeutesteigerung an 12 auf $58 \%$. Trotz dieser relativ guten Ausbeute konnten wir keine Spur einer Austauschreaktion in para-Position feststellen. Bei der von Finger et al. ${ }^{6}$ mit nur 10\% verlaufenden Austauschreaktion der zum Fluor ortho-ständigen NitroGruppe wäre eine kleine Menge eines zweiten Isomers nur schwierig nachweisbar gewesen.

Die hohe Selektivität bei der Umsetzung von 11 zu 12 in Gegenwart des Säurechlorids 13 könnte mit einer Begünstigung der ortho-Substitution durch den stark induktiven Effekt des Fluor-Atoms erklärt werden, der sich besonders bei schwachen Nucleophilen - wie dem Fluorid - bemerkbar macht. So wurde bei der nucleophilen Substitution aromatischer Nitro-Gruppen im (Perfluorisopropyl)-2,4-dinitrobenzol mit Methanolat ${ }^{18 a}$ sowie im 2,4-Dinitrobenzonitril mit Piperidin ${ }^{18 b)}$ eine Reaktion nur in 4-Stellung gefunden; bei der Nitrit-Substitution in 3-(2,4-Dinitrophenyl)1,2,5-thiadiazol mit Alkoholen/ $\mathrm{KOH} / \mathrm{H}_{2} \mathrm{O}^{19 a)}$ sowie in 2,4Dinitrotoluol mit Lithiumethanthiolat ${ }^{19 b}$ wurde nur eine Reaktion in 2-Position erhalten. In dem letztgenannten Beispiel wird die ortho-Selek tivität mit der aus sterischen Gründen geringeren Mesomeriebeteiligung der ortho-NitroGruppe an dem zu bildenden Meisenheimer-Komplex erklärt.

Asymmetrische Biphenyle sind mit den üblichen Darstellungsmethoden für Biphenyle nur in sehr mäßigen Ausbeuten erhältlich; 4-Fluor-4'-nitrobiphenyl (15) $z$. B. wurde ausgehend von 4,4'-Dinitrobiphenyl (14) über das 4-Amino-4'nitrobiphenyl, dessen Diazotierung und anschließende Thermolyse des gebildeten Diazoniumtetrafluoroborats in nur 14proz. Ausbeute hergestellt ${ }^{20)}$. Wir haben deshalb die Möglichkeit eines Nitrit/Fluorid-Austauschs auch an Nitrobiphenylen untersucht.

Schema 5

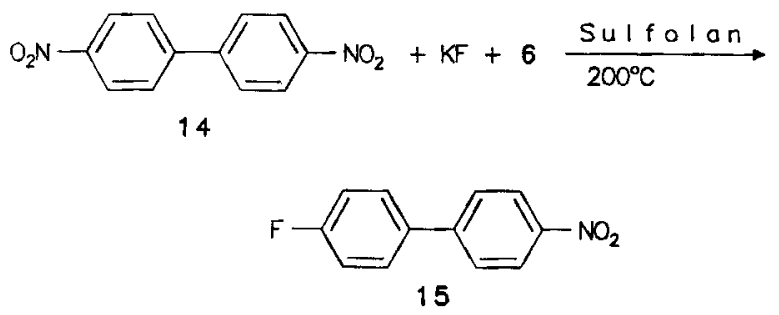

Bei der Umsetzung von 14 mit Kaliumfluorid in Sulfolan bei $200^{\circ} \mathrm{C}$ unter Zugabe von 6 konnten wir nach 32 Stunden bei $200{ }^{\circ} \mathrm{C} 15$ mit nur $9 \%$ neben $88 \%$ Ausgangsprodukt 14 gaschromatographisch nachweisen; in Gegenwart von Kro- nenether erhöhte sich nach 23 Stunden die Ausbeute von 15 auf $15 \%$.

Dieses Verfahren befriedigt von den Ausbeuten her zwar keineswegs, es ist jedoch den bisherigen Darstellungsmethoden überlegen und bestätigt literaturbekannte Ergebnisse über nucleophile Substitutionsreaktionen an 4-Halogen-4'nitro-substituierten Biphenylen ${ }^{21)}$, die - bedingt durch die Verdrillung der beiden Phenyl-Ringe gegeneinander und die dadurch herabgesetzte Mesomeriewechselwirkung - wesentlich langsamer reagieren als z. B. 4-Halogennitrobenzole.

Wir danken der Deutschen Forschungsgemeinschaft und dem Fonds der Chemischen Industrie für die Unterstützung dieser Arbeit. Herrn cand. chem. D. Weller danken wir für engagierte Mitarbeit.

\section{Experimenteller Teil}

${ }^{1}$ H-NMR: T 60, A 60 und EM 360 der Firma Varian sowie WP 80 und HX 90 der Firma Bruker; Tetramethylsilan als interner Standard. - Säulenchromatographie: Glassäulen verschiedener Größe, gefüllt mit Kieselgel S, Korngröße 0.040-0.063 mm (Fa. Riedel-de Haën). - Gaschromatographie: a) $5700 \mathrm{~A}$ und $5710 \mathrm{~A}$ der Firma Hewlett- Packard; Integrator: Varian Aerograph 477 und Spectraphysics Minigrator; $30 \mathrm{ml} / \mathrm{min}$ Rein-Stickstoff; Glaskapillare $2.30 \times 2 \mathrm{~mm}$ OV 17/10\% auf Chromosorb W. b) Carlo Erba Fractovap GI mit FID; Integrator: Spectraphysics Minigrator; 0.7 bar Helium; Glaskapillare $20 \mathrm{~m} \times 2 \mathrm{~mm}$, Phase SE 52 .

$\mathrm{KF}, \mathrm{CsF}$ : Die Salze wurden 4 mal jewcils $8 \mathrm{~h}$ in der Kugelmühle gemahlen und $8 \mathrm{~h}$ bei $150^{\circ} \mathrm{C} / 100$ Torr im Trockenschrank über Phosphorpentoxid, anschließend $24 \mathrm{~h}$ bei $300^{\circ} \mathrm{C} / 5 \times 10^{-2}$ Torr im Hochvakuumtrockenschrank getrocknet und dann noch $10 \mathrm{~h}$ in der Kugelmühle gemahlen.

1) Umsetzungen von 1,3-Dinitrobenzol (1) mit $K F$ in N-Methyl-2pyrrolidon. - a) Nach Lit. ${ }^{7)}$ : Man rührt in einem mit fächelnder Flamme im trockenen Stickstoff-Strom ausgeheizten 250-ml-Kolben mit aufgesetztem Rückflußkühler $48 \mathrm{~h}$ bei $180^{\circ} \mathrm{C}$ unter Stickstoff $27.0 \mathrm{~g}(0,16 \mathrm{~mol}) 1$ und $81.0 \mathrm{~g}(1.39 \mathrm{~mol}) \mathrm{KF}$ in $101 \mathrm{ml} N$ Methyl-2-pyrrolidon. Nach Wasserdampfdestillation wird die wäßrige Phase $4 \mathrm{mal}$ mit $150 \mathrm{ml}$ Diethylether extrahiert, die Ether-Extrakte werden mit Calciumchlorid getrocknet und nach Entfernen des Lösungsmittels im Rotationsverdampfer destilliert; Ausb. $5.0 \mathrm{~g}$ (22\%) 3-Fluornitrobenzol (2), Sdp. $53.5^{\circ} \mathrm{C} / 5 \times 10^{-2}$ Torr (Lit. ${ }^{22)}$ $86^{\circ} \mathrm{C} / 19$ Torr).

b) Wie unter 1a) beschrieben; jedoch wurden jeweils $6.50 \mathrm{~g}(38.7$ mmol) 1 mit $6.73 \mathrm{~g}$ (116.1 mmol) $\mathrm{KF}$ in $13 \mathrm{ml} N$-Methyl-2-pyrrolidon bei $170^{\circ} \mathrm{C}$ gerührt. Nach Abkühlen auf Raumtemperatur wurde Hydrochinon-dimethylether (als Standard) zugegeben, mit $75 \mathrm{ml}$ Diethylether extrahiert, der Extrakt $2 \mathrm{mal}$ mit jeweils $75 \mathrm{ml}$ Wasser gewaschen und mit Magnesiumsulfat getrocknet. Die Ausbeuten wurden gaschromatographisch nach Lit. ${ }^{23)}$ bestimmt.

\begin{tabular}{rlcl}
$\begin{array}{c}\text { nach } \\
{[\mathrm{h}]}\end{array}$ & $\mathbf{1}$ & $\begin{array}{c}\text { Ausbeuten }(\%) \\
\mathbf{2}\end{array}$ & $\mathbf{5}$ \\
\hline 3 & 86 & 12 & \\
24 & 53 & 13 & 11 \\
36 & 34 & 17 & 15 \\
42 & 36 & 13 & 14 \\
\hline 72 & 12 & \\
\hline
\end{tabular}


c) Wie unter 1b) beschrieben wurden $168.0 \mathrm{~g}(1.0 \mathrm{~mol}) 1 \mathrm{mit}$

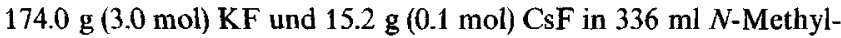
2-pyrrolidon $15 \mathrm{~h}$ auf $170^{\circ} \mathrm{C}$ erhitzt. Die Hälfte des Reaktionsgemisches wurde destilliert, Ausb. $38.0 \mathrm{~g}(27 \%) 2$ und $73.92 \mathrm{~g}(44 \%)$ 1, Sdp. $120^{\circ} \mathrm{C} / 5 \times 10^{-2}$ Torr (Lit. ${ }^{24} 167^{\circ} \mathrm{C} / 14$ Torr). Der Destillationsrückstand wurde bei $140^{\circ} \mathrm{C} / 10^{-3}$ Torr fraktionierend sublimiert, Ausb. $6.5 \mathrm{~g}$ (50\%) 3,3'-Dinitrodiphenylether (5), Schmp. $123^{\circ} \mathrm{C}$.

\section{5: $\mathrm{C}_{12} \mathrm{H}_{8} \mathrm{~N}_{2} \mathrm{O}_{5}(260.05) \quad$ Ber. C 55.39 H $3.09 \mathrm{~N} 10.77$ Gef. C 55.40 H 2.99 N 10.84}

Die zweite Hälfte des Reaktionsgemischs wurde an Kieselgel chromatographiert, Ausb. $36.7 \mathrm{~g}(26 \%) 2$ (mit Tetrachlormethan als Eluens); $6.0 \mathrm{~g}(9 \%) 5$ (1. Fraktion) und $72.24 \mathrm{~g}(43 \%) 1$ (2. Fraktion) [mit Tetrachlormethan/Essigsäure (10:1) als Eluens].

2) Umsetzung von 1,3-Dinitrobenzol (1) mit KF mit höherem Wassergehalt: Wie unter $1 \mathrm{~b}$ ) beschrieben jedoch mit $\mathrm{KF}$, das nach 6 stdg. Mahlen in der Kugelmühle $6 \mathrm{~h}$ bei $150^{\circ} \mathrm{C} / 100$ Torr im Trockenschrank getrocknet und anschließend nochmals $6 \mathrm{~h}$ in der Kugelmühle gemahlen wurde.

\begin{tabular}{cccc}
$\begin{array}{c}\text { nach } \\
{[\mathrm{h}]}\end{array}$ & $\mathbf{1}$ & $\begin{array}{c}\text { Ausbeuten }(\%)^{\mathrm{a})} \\
\mathbf{2}\end{array}$ & $\mathbf{5}$ \\
\hline 24 & 52 & 13 & 12 \\
30 & 39 & 13 & 14 \\
48 & 26 & 17 & 20 \\
54 & 12 & 14 & 17
\end{tabular}

a) Gaschromatographisch bestimmt.

3) Umsetzungen von 1-Fluor-3-nitrobenzol (2) mit $\mathrm{KNO}_{2}$ : Man rührt wie unter 1 b) beschrieben $3.0 \mathrm{~g}(21.3 \mathrm{mmol}) 2$ und $1.8 \mathrm{~g} \mathrm{(21.2}$ mmol) $\mathrm{KNO}_{2}$ in $7.1 \mathrm{ml} \mathrm{N}$-Methylpyrrolidon bei $170^{\circ} \mathrm{C}$.

\begin{tabular}{cccc}
$\begin{array}{c}\text { nach } \\
{[\mathrm{h}]}\end{array}$ & $\mathbf{1}$ & $\begin{array}{c}\text { Ausbeuten }(\%)^{\text {a) }} \\
\mathbf{2}\end{array}$ & $\mathbf{5}$ \\
\hline 2 & 87 & 11 & 6 \\
6 & 79 & 15 & 7 \\
12 & 65 & 21 & 10 \\
18 & 48 & 18 & 14 \\
24 & 42 & 19 & 13 \\
30 & 36 & 18 & 12 \\
36 & 26 & 13 & 10
\end{tabular}

a) Gaschromatographisch bestimmt.

4) Umsetzung von 1,3-Dinitrobenzol (1) mit $\mathrm{KF} / \mathrm{CsF}$ in Gegenwart von $\left(\mathrm{NH}_{4}\right)_{2} \mathrm{SO}_{4}$ : In einem mit fächelnder Flamme im trockenen Stickstoff-Strom ausgeheizten 50-ml-Kolben mit aufgesetztem Rückflußkühler wurden $6 \mathrm{~h}$ bei $170^{\circ} \mathrm{C}$ unter Stickstoff $6.50 \mathrm{~g} \mathrm{(38.7}$ $\mathrm{mmol}) 1,6.75 \mathrm{~g}(116.1 \mathrm{mmol}) \mathrm{KF}, 15.35 \mathrm{~g}(116.2 \mathrm{mmol})\left(\mathrm{NH}_{4}\right)_{2} \mathrm{SO}_{4}$, $590.0 \mathrm{mg}$ (3.9 mmol) $\mathrm{CsF}$ und $13 \mathrm{ml} \mathrm{N}$-Methyl-2-pyrrolidon gerührt. Das Reaktionsgemisch wurde dann mit $75 \mathrm{ml}$ Diethylether extrahiert, der Extrakt mit $75 \mathrm{ml}$ Wasser gewaschen, mit Calciumchlorid getrocknet, im Rotationsverdampfer eingeengt und der Rückstand an Kieselgel mit Dichlormethan chromatographiert; Ausb. $0.69 \mathrm{~g} \mathrm{(13 \% )} \mathrm{3-Nitranilin,} \mathrm{Schmp.} 114^{\circ} \mathrm{C}$ (Lit. ${ }^{25)} 114^{\circ} \mathrm{C}$ ).

5) Umsetzung von $\mathrm{KNO}_{2}$ mit Phthaloyldichlorid (6): In einem ausgeheizten 50-ml-Zweihalskolben (mit Gummiseptum und Rückflußkühler) wurden unter Stickstoff $1.64 \mathrm{~g}(19.3 \mathrm{mmol}) \mathrm{KNO}_{2}$ eingewogen. Nach Abschließen der Apparatur gegen Feuchtigkeit mit einem mit Silicon-Öl gefüllten Gasblasenzähler wurden $3.93 \mathrm{~g}$ (19.3 mmol) 6 und $13 \mathrm{ml}$ Sulfolan durch das Septum zugespritzt, dann wurde das Reaktionsgemisch $2.5 \mathrm{~h}$ bei $180^{\circ} \mathrm{C}$ mit einem Rührmagnet gerührt und anschließend mit $20 \mathrm{ml}$ Wasser verdünnt. Der Nachweis von eventuell noch vorhandenem Nitrit (Zugabe von Amidosulfonsäure) verlief negativ, der ausgefallene Niederschlag wurde abgesaugt und über Phosphorpentoxid getrocknet; Ausb. $2.6 \mathrm{~g}(90 \%)$ Phthalsäureanhydrid (7), Schmp. $130^{\circ} \mathrm{C}\left(\right.$ Lit. $\left.^{26)} 131.6^{\circ} \mathrm{C}\right)$.

6) Umsetzungen von 1,3-Dinitrobenzol (1) mit $K F$ in Gegenwart von Phthaloyldichlorid (6): a) Wie unter 5) beschrieben wurden unter Stickstoff $3.25 \mathrm{~g}(19.35 \mathrm{mmol}) \mathbf{1}, 6.73 \mathrm{~g}(116.0 \mathrm{mmol}) \mathrm{KF}$ und 294.0 $\mathrm{mg}(1.9 \mathrm{mmol}) \mathrm{CsF}$ eingewogen und $3.93 \mathrm{~g}(19.3 \mathrm{mmol}) 6$ und $13 \mathrm{ml}$ Sulfolan durch das Gummiseptum zugespritzt (Reaktionstemperaturen und -zeiten siehe Tab. 1). Nach Zugabe von $50 \mathrm{ml}$ Wasser wurde das Reaktionsgemisch $2 \mathrm{mal} \mathrm{mit} \mathrm{jeweils} 50 \mathrm{ml}$ Diethylether extrahiert, der Ether-Extrakt mit Magnesiumsulfat getrocknet, im Rotationsverdampfer eingeengt, der Rückstand mit Dichlormethan/ Tetrachlormethan $(1: 2)$ aufgenommen und an Kieselgel chromatographiert. Produkte und Ausbeuten wurden gaschromatographisch durch Koinjektion mit Vergleichssubstanzen bestimmt (siehe Tab. 1).

b) Wie unter 6a) beschrieben, jedoch ohne CsF mit $5.61 \mathrm{~g} \mathrm{(96.7}$ mmol) KF. Nach Reaktionsende wurden $1.0 \mathrm{~g}$ Hydrochinon-dimethylether (als Standard) zugegeben, anschließend wurde das Reaktionsgemisch mit $50 \mathrm{ml}$ Wasser gewaschen, die organische Phase abgetrennt, mit Magnesiumsulfat getrocknet, im Rotationsverdampfer eingeengt, der Rückstand in $50 \mathrm{ml}$ Dichlormethan gelöst und gaschromatographisch ausgewertet (siehe Tab. 1).

c) Wie unter 6b) beschrieben, jedoch mit insgesamt $5.95 \mathrm{~g}(29.3$ mmol) 6. Nach Einwiegen von $3.25 \mathrm{~g} 1$ und $5.61 \mathrm{~g} \mathrm{KF}$ unter Stickstoff wurden $20 \mathrm{ml}$ Sulfolan und $3.93 \mathrm{~g}(19.3 \mathrm{mmol}) 6$ zugespritzt, dann wurde $24 \mathrm{~h}$ bei $200^{\circ} \mathrm{C}$ gerührt. Nach Abkühlen auf $140^{\circ} \mathrm{C}$ wurden erneut $1.46 \mathrm{~g}(7.1 \mathrm{mmol}) 6$ zugespritzt, dann wurde $15 \mathrm{~min}$ bei $140^{\circ} \mathrm{C}$ und $24 \mathrm{~h}$ bei $200^{\circ} \mathrm{C}$ gerührt. Derselbe Vorgang wurde nach $\mathrm{Abkühlen}$ auf $140^{\circ} \mathrm{C}$ und Zuspritzen von weiteren $0.56 \mathrm{~g} \mathrm{(2.7}$ mmol) 6 nochmals wiederholt, dann wurde das Reaktionsgemisch auf Raumtemperatur abgekühlt. Aufarbeitung und Auswertung erfolgten wie unter $6 \mathrm{~b}$ ) beschrieben (siehe Tab. 1).

d) Wie unter 6c) beschrieben, jedoch mit wechselnden Mengen KF [4.48 g (77.2 mmol) bzw. $6.73 \mathrm{~g}(116.0 \mathrm{mmol})]$ (siehe Tab. 1).

e) Präparative Darstellung von 1-Fluor-3-nitrobenzol (2): Wie unter 6 c) beschrieben, jedoch wurde der 10 fache Ansatz in einem ausgeheizten 500-ml-Dreihalskolben (mit Gummiseptum, Rückflußkühler und KPG-Rührer) bei gleichen Reaktionszeiten und -temperaturen durchgeführt. Nach insgesamt 72 stdg. Reaktionszeit wurde auf Raumtemperatur abgekühlt und 2 bei 0.05 Torr in eine mit Trockeneis gekühlte Vorlage kondensiert; Ausb. $15.3 \mathrm{~g} \mathrm{(56 \% )}$ (gaschromatographisch identifiziert durch Koinjektion von Vergleichssubstanz). Der Rückstand wurde bei $40^{\circ} \mathrm{C} / 0.05$ Torr destilliert, das erhaltene Destillat (ein Gemisch aus 2 und Sulfolan) mit $20 \mathrm{ml}$ Diethylether verdünnt und mit $50 \mathrm{ml}$ Wasser gewaschen. Nach Trocknen der organischen Phase mit Magnesiumsulfat und Entfernen des Ethers im Rotationsverdampfer blieben weitere $3.8 \mathrm{~g}$ $(14 \%) 2$ zurück; Gesamtausb. $19.1 \mathrm{~g}(70 \%)$. Der Destillationsrückstand wurde bei $80^{\circ} \mathrm{C} / 0.05$ Torr destilliert, aus dem übergehenden Gemisch mit $0.1 \mathrm{~N} \mathrm{HCl} 7$ gefällt, abgesaugt, mit Wasser gewaschen und im Exsikkator über Phosphorpentoxid getrocknet; Ausb. $30.0 \mathrm{~g}$ (70\%), Schmp. $130^{\circ} \mathrm{C}$.

7) Umsetzungen von 1,3,5-Trinitrobenzol (8) mit $K F$ in Sulfolan: Jeweils $2.06 \mathrm{~g}(9.7 \mathrm{mmol}) 8$ und $3.93 \mathrm{~g}(67.7 \mathrm{mmol}) \mathrm{KF}$ wurden a) mit $3.93 \mathrm{~g}(19.35 \mathrm{mmol})$, b) mit $1.96 \mathrm{~g}(9.7 \mathrm{mmol}) 6$ und c) ohne 6 in jeweils $6.5 \mathrm{ml}$ Sulfolan bei $180^{\circ} \mathrm{C}$ wie unter $6 \mathrm{~b}$ ) beschrieben umgesetzt und aufgearbeitet (Ergebnisse siehe Tab. 2). 
8) 1-Fluor-3,5-dinitrobenzol (9): $6.18 \mathrm{~g} \mathrm{(29.0} \mathrm{mmol)} \mathrm{8,8.41} \mathrm{g} \mathrm{(145.1}$ mmol) $\mathrm{KF}$ und $5.89 \mathrm{~g}(29.0 \mathrm{mmol}) 6$ wurden in $19.5 \mathrm{ml}$ Sulfolan $53 \mathrm{~min}$ bei $180^{\circ} \mathrm{C}$ gerührt. Nach Abkühlen und Zugeben von $100 \mathrm{ml}$ Diethylether wurde das Reaktionsgemisch $2 \mathrm{mal}$ mit $50 \mathrm{ml}$ Wasser gewaschen, die organische Phase abgetrennt, mit Magnesiumsulfat getrocknet und im Rotationsverdampfer eingeengt. Der Rückstand wurde in Dichlormethan gelöst und an Kieselgel chromatographiert; Ausb. $4.57 \mathrm{~g}(85 \%)$, Schmp. $43^{\circ} \mathrm{C}$ (Lit. ${ }^{27)} 43.8^{\circ} \mathrm{C}$ ).

9) 1,3-Difluor-5-nitrobenzol (10): $15.0 \mathrm{~g}(70.4 \mathrm{mmol}) \mathbf{8}, 28.59 \mathrm{~g}$ (492.1 mmol) $\mathrm{KF}$ und $28.59 \mathrm{~g}$ (140.8 mmol) 6 wurden in $47 \mathrm{ml}$ Sulfolan $12 \mathrm{~h}$ bei $180^{\circ} \mathrm{C}$ gerührt. Nach Abkühlen und zweimaliger Extraktion des Reaktionsgemischs mit jeweils $100 \mathrm{ml}$ Diethylether wurde der Extrakt mit Magnesiumsulfat getrocknet, im Rotationsverdampfer eingeengt, der Rückstand mit $50 \mathrm{ml}$ Diethylether versetzt und ausgefallenes 7 abgesaugt; Ausb. 15.63 g (75\%). Das Filtrat wurde im Rotationsverdampfer eingeengt und der Rückstand destilliert; Ausb. $7.83 \mathrm{~g} \mathrm{(70 \% ),} \mathrm{Sdp.} 70^{\circ} \mathrm{C} / 15$ Torr $\left(\right.$ Lit. $^{28)} 78-79^{\circ} \mathrm{C} /$ 20 Torr).

10) 1,2-Difluor-4-nitrobenzol (12): a) In einem unter trockenem Stickstof ausgeheizten 250-ml-Dreihalskolben (mit Rührmagnet, Rückfluß3kühler, Tropftrichter und Gummiseptum) wurden unter Stickstoff $28.06 \mathrm{~g}$ (483.0 mmol) KF und $1.47 \mathrm{~g}(9.7 \mathrm{mmol}) \mathrm{CsF}$ eingewogen und $19.65 \mathrm{~g}(96.8 \mathrm{mmol}) 6$ sowie $50 \mathrm{ml}$ Sulfolan durch das Septum zugespritzt. Nach Abschließen der Apparatur mit einem mit Siliconöl gefüllten Gasblasenzähler wurde das Reaktionsgemisch auf $190^{\circ} \mathrm{C}$ erhitzt, dann wurden $18.60 \mathrm{~g}(100 \mathrm{mmol}) 11$ innerhalb 17 h unter Rühren zugetropft. Nach Abkühlen wurde das Reaktionsgemisch mit $150 \mathrm{ml}$ Diethylether verdünnt, $3 \mathrm{mal} \mathrm{mit} \mathrm{jeweils}$ $75 \mathrm{ml}$ Wasser gewaschen, die organische Phase abgetrennt, mit Magnesiumsulfat getrocknet, im Rotationsverdampfer eingeengt und der Rückstand destilliert; Ausb. $5.64 \mathrm{~g} \mathrm{(35 \% ),} \mathrm{Sdp.} 76-80^{\circ} \mathrm{C} /$ 10 Torr (Lit. ${ }^{6)} 76-80^{\circ} \mathrm{C} / 11$ Torr).

b) $1.80 \mathrm{~g}(9.67 \mathrm{mmol}) 11,3.37 \mathrm{~g}(58.1 \mathrm{mmol}) \mathrm{KF}$ und $3.15 \mathrm{~g}(9.6$ mmol) 13 in $13 \mathrm{ml}$ Sulfolan wurden 4 h bei $200^{\circ} \mathrm{C}$ wie unter $6 \mathrm{~b}$ ) beschrieben umgesetzt und aufgearbeitet; Ausb. $58 \% 12$ sowie $11 \%$ nicht umgesetztes 11 (gaschromatographisch bestimmt).

11) Umsetzung von 4,4'-Dinitrobiphenyl (14) mit $K F$ : a) $4.72 \mathrm{~g}$ (19.35 mmol) $14,5.61 \mathrm{~g}(96.7 \mathrm{mmol}) \mathrm{KF}$ und $3.93 \mathrm{~g}(19.35 \mathrm{mmol}) 6$ wurden in $20 \mathrm{ml}$ Sulfolan $3 \mathrm{~h}$ bei $200^{\circ} \mathrm{C}$ wie unter $6 \mathrm{~b}$ ) beschrieben umgesetzt und aufgearbeitet; Ausb. 9\% 4-Fluor-4'-nitrobiphenyl (15) sowie $88 \%$ nicht umgesetztes 14 (gaschromatographisch bestimmt).

b) Wic unter a) beschrieben, jedoch in Gegenwart von $0.35 \mathrm{~g}$ Dibenzo-18-krone-6 und 23stdg. Reaktionszeit bei $200^{\circ} \mathrm{C}$; Ausb. $15 \% 15$ sowie $82 \%$ nicht umgesetztes 14 (gaschromatographisch bestimmt).

\section{CAS-Registry-Nummern}

1: 99-65-0 / 2: 402-67-5 / 5: 38490-83-4 / 6: 88-95-9 / 7: 85-44-9 / 8: $99-35-4 / 9: 369-18-6 /$ 10: 2265-94-3/11: 70-34-8/12: 369 . 34-6/13: $7710-20-5 / 14: 1528-74-1 / 15: 398-24-3 / \mathrm{KF}: 7789$ 23-3 / CsF: 13400-13-0/ $\mathrm{KNO}_{2}: 7758-09-0 /\left(\mathrm{NH}_{4}\right)_{2} \mathrm{SO}_{4}: 35089-90-8$

i) 1a) W. Streicher, Diplomarbeit, Univ. Stuttgart, 1981, - ${ }^{\text {b) }}$ W. Streicher, Teil der Dissertation, Univ. Stuttgart, 1983.
2) 2a) G. Balz, G. Schiemann, Ber. Dtsch. Chem. Ges. 60 (1927) 1186. - 2b) E. Forche in Methoden der Organischen Chemie (Houben-Weyl-Müller), 4. Aufl., Bd. V/3, S. 214, Thieme, Stuttgart 1962. - ${ }^{2 c)} \mathrm{R}$. Pütter in Methoden der Organischen Chemie (Houben-Weyl-Müller), 4. Aufl., Bd. X/3, S. 37, Thieme, Stuttgart 1965. - ${ }^{2 d)}$ K. H. Saunders, R. L. M. Allen in Aromatic Diazo Compounds (E. Arnold, Hrsg.), 3. Aufl., S. 744, Thomson Lithio Ltd., East Kilbride, Scotland 1985.

3) 3a) J. F. Bunnett, R. E. Zahler, Chem. Rev. 49 (1951) 273. - ${ }^{3 b j}$ F. Pietra, D. Vitali, J. Chem. Soc., Perkin Trans 2, 1972, 385. 3c) G. Bartoli, P. E. Todesco, Acc. Chem. Res. 10 (1977) 125.

4) ${ }^{4 a)}$ R. E. Parker, T. O. Read, J. Chem. Soc. 1962, 3149. - 4b) J. Hayami, M. Asahi, R. Tamura, N. Ono, Bull. Inst. Chem. Res., Kyoto Univ. 58 (1980) 222 [Chem. Abstr. 94 (1981) 46372 s].

5) 5a) J. R. Beck, Tetrahedron 34 (1978) 2057. - 5b) H. Guo, Hua Hsue Tung Pao 1983, 38-46, 27 [Chem. Abstr. 99 (1983) $139396 \mathrm{w}]$.

6) G. C. Finger, C. W. Kruse, J. Am. Chem. Soc. 78 (1956) 6034.

7) G. Bartoli, A. Latrofa, F. Naso, P. E. Todesco, J. Chem. Soc., Perkin Trans. 1, 1972, 2671.

8) S. E. Morgan, D. M. Rackham, B. P. Swann, S. P. Turner, Tetrahedron Lett. 1978, 4837.

9! N. Kornblum, L. Cheng, R. C. Kerber, M. M. Kestner, B. N. Newton, H. W. Pinnick, R. G. Smith, P. A. Wade, J. Org. Chem. 41 (1976) 1561

10) 10a) D. H. Rosenblatt, W. H. Dennis, Jr., R. D. Goodin, J. Am. Chem. Soc. 95 (1973) 2133. - 10b) T.J. Broxton, D. M. Muir, A. J. Parker, J. Org. Chem. 40 (1975) 3230. - ${ }^{10 b)}$ V. V. Plakhtinskii, V. A. Ustinw, G. S. Mironov, Izv. Vyssh. Uchebn. Zaved., Khim. Khim. Tekhnol. 28 (1985) 3 [Chem. Abstr. 102 (1985) 203358v].

${ }^{11)}$ R. L. Markezich, O. S. Zamek, P. E. Donahue, F. J. Williams, J. Org. Chem. 42 (1977) 3435.

12) Lit. ${ }^{2 b)}$, S. 235.

13) 13a) G. Schiemann, J. Prakt. Chem. 140 (1934) 97. - ${ }^{136)}$ G. C. Finger, F. H. Reed, J. L. Finnerty, J. Am. Chem. Soc. 73 (1951) 153.

14) H. Zahn, A. Würz, Angew. Chem. 63 (1951) 147.

15) BASF A. G. (F. Effenberger, W. Streicher, Erf.) Ger. Offen. DE 3,400,418 (Cl. C07C79/12), 18. Juli 1985, Appl. 7. Jan. 1984. [Chem. Abstr. 104 (1968) P $148476 \mathrm{w}]$.

16) T6a) J. J. Blanksma, Recl. Trav. Chim. Pays-Bas 65 (1946) 329. ${ }^{16 \mathrm{~b})}$ F. Weigand, Chem. Ber. 84 (1951) 109.

17) 17at E. Bergmann, S. Berkovic, R. Ikan, J. Am. Chem. Soc. 78 (1956) 6037. - 13b) Eastman Kodak Co. (J. G. McNally, J. R. Byers, Jr., Erf.), U.S.Pat. 2,391,179 (18. Dez. 1945) [Chem. Abstr. 40 (1946) $\left.2635^{2}\right]$.

18) 18a) N. Ishikawa, Y. Inoue, K. Kitagawa, Nippon Kagaku Zasshi 91 (1970) 742 [Chem. Abstr. 73 (1970) 109401 q]. - ${ }^{18 b)}$ C. W. N. Holmes, J. D. Loudon, J. Chem. Soc. 1940, 1521

19) ${ }_{19}$ A. De Munno, V. Bertini, G. Denti, Int. J. Sulfur Chem., Part A. 2 (1972) 25 [Chem. Abstr. 77 (1972) 126514d]. - 196) F. Benedetti, D. R. Marshall, C. J. M. Stirling, J. L. Leng, J. Chem. Soc., Chem. Commun. 1982, 918.

20) 20a) E. E. J. Marler, E. E. Turner, J. Chem. Soc. 1931, 1359. ${ }^{20 b)}$ R. Belcher, A. J. Nutten, W. I. Stephen, J. Chem. Soc. 1953, 1334.

21) 21a) C. Dell'Erba, G. Guanti, G. Carbarino, Tetrahedron 27 (1971) 1807. - ${ }^{21 b}$ E. Berliner, B. Newman, T. M. Riaboff, J. Am. Chem. Soc. 77 (1955) 478. - 21c) D. J. Byron, G. W. Gray, R. C. Wilson, J. Chem. Soc. C, 1966, 831.

22) R. C. Weast, M. J. Astle, CRC Handbook of Chemistry and Physics, CRC Press, Inc., Boca Raton, Florida: 22a) 63. Aufl. , C-137, $1982-1983 ;{ }^{22 b)}$ 57. Aufl., C-165, 1976-1977.

${ }^{23)} \mathrm{R}$. Kaiser in Chromatographie in der Gasphase, Bd. 4, S. 206, Bibliographisches Institut, Mannheim 1965.

24) Lit. 22a), C-136.

25) Lit. ${ }^{22 a)}$ C-96.

26) Lit. ${ }^{22 a)}, \mathrm{C}-454$.

27) B. Bye, J. Chem. Soc. 1954, 3091

${ }^{28)}$ G. C. Finger, F. H. Reed, D. M. Burness, D. M. Forst, R. R. Blough, J. Am. Chem. Soc. 73 (1951) 145.

$168 / 90]$ 